\title{
INTERNET OF THINGS (IoT) BASED SWITCHBOX USING MQTT PROTOCOL
}

\author{
Neha Agarwal ${ }^{1}$, Sunhera Paul ${ }^{2}$, Parth Gujar ${ }^{3}$, Viraj Gite ${ }^{4}$ \\ ${ }^{1}$ Student, Computer Department, Maharashtra Institute of Technology, Maharashtra, India \\ ${ }^{2}$ Student, Computer Department, Maharashtra Institute of Technology, Maharashtra, India \\ ${ }^{3}$ Student, Computer Department, Maharashtra Institute of Technology, Maharashtra, India \\ ${ }^{4}$ Student, Computer Department, Maharashtra Institute of Technology, Maharashtra, India
}

\begin{abstract}
Internet of Things (IoT) can be defined as the network of physical objects or things embedded with electronics, software, sensors, and connectivity to enable "things" to exchange data with the operator and/or other connected devices. IoT devices are very useful to monitor and control the mechanical, electrical and electronic systems used in various buildings (e.g. public and private, industrial, institutions, or residential). Home automation systems, like other building automation systems, can be typically used to control lighting, heating, ventilation, air conditioning, appliances, communication systems, entertainment and home security devices. This is basically used to improve convenience, comfort, energy efficiency, and security. An IoT based home automation even though realizable today, haven't made the lives of masses easier yet due the way it is implemented till date. The reason is that the products have remained as a complete set of units which lack modularity and also exclude the legacy appliances from connectivity to the smart ecosystem. Also, the costs of such products have been so high that the masses cannot afford such systems. This paper presents a highly modular, affordable and a smart system that is the IoT enabled switchbox which is rich in features and will enable the masses to live a luxurious life. This switchbox would be facilitated with internet connectivity allowing switching on/off and also increasing/decreasing the intensity of the appliances connected to it remotely.
\end{abstract}

Keywords: Home Automation System, Internet of Things(IoT), Cloud computing, Wi-Fi network

\section{INTRODUCTION}

Internet of Things (Iot) is a network or connection of physical objects or "things" embedded with electronics, software, sensors, making these objects capable of collection of data. The basic idea of an IoT based switchbox is to broaden the working of a normal switchbox in such a way that the switches can be accessed pervasively. The IoT based switchbox is anticipated to enable the user to access the switchbox remotely which is made possible through an android application to interact with the devices through only basic internet access.

The proposed product is entirely based on the concept of IoT that facilitates the connection and control of product remotely. The implementation of IoT makes a great deal of emphasis on giving internet connectivity to the device, which is implemented using internet on chip microcontroller. This ability to access and use the internet is used to connect the switchbox to the mobile app/web app which will be the control point of user.

\section{NEED OF THE PROJECT}

The major drive of this project lies in enhancing the lifestyle of the users. Its gives a cost effective solution to replace the existing toggle switch boxes with the facility of accessing them from any part of the world only by touch through an android application. This switchbox has modular approach as one of its important and key features which enables in reducing the cost of integrating new devices in the existing switchbox. It also greatly reduces the human effort by providing an easy access via an application which is very easy to operate even for naive users.

Goal and Objectives:

1. To develop a system for remote as well as local access of electronic devices which is controlled by a switchbox installed at home and uses Internet of Things to provide remote access to connected devices.

2. To replace the existing toggle and push button switchboxes with a modular version which can be accessed locally using touch switches and remotely using internet via an android application.

\section{PROPOSED WORK}

The basic working of the proposed product is explained below:

1. The product is an IoT based switchbox which takes inputs as the specific switch from all available and installed boxes and the appropriate switch function for required device.

2. Inputs are the selection of switchbox, then switch from that switchbox and finally functionality required for that switch. eg switch ON/OFF, eg intensity of device INCREASE/REDUCE. 
3. The proposed product makes changes in the current state of selected device according to available and selected functionality.

eg. intensity INCREASE/REDUCE is available for only certain devices like fan and other devices like tubelight have only functionality to switch ON/OFF.

\section{ARCHITECTURAL DESIGN}

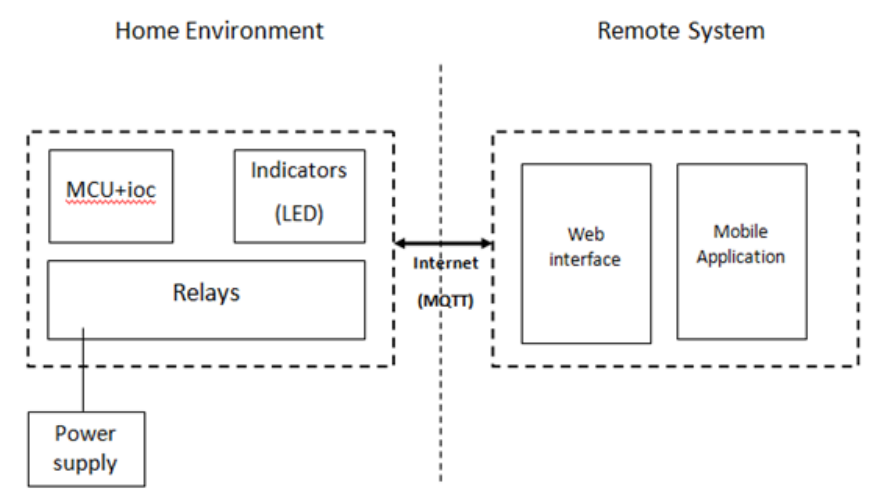

Fig. Architectural Diagram

Description of the components:

\section{MICROCONTROLLER}

A microcontroller can basically be described as a small computer (SoC) on a single IC which contains a processor core, memory, and programmable input/output peripherals. Program memory in the various forms of Ferroelectric RAM, NOR flash or OTP ROM is also included on chip. Also, a small amount of RAM is included on the chip. Microcontrollers are basically designed for embedded applications as they are more efficient for embedded applications as compared to general purpose applications and computers where microprocessors work better.

\section{RELAYS}

A relay can be described in broad and general terms as an electrically operated switch. Many relays use an electromagnet to operate a switch mechanically. But it is possible to use other operating principles such as solid-state relays. Relays are used where several circuits must be controlled by one signal. They can also be made useful where it is required to control a circuit by a low powered signal.

\section{MOBILE APPLICATION}

A mobile application is a computer program designed to run on devices which have mobility eg. smart phones and tablet computers. Mobile applications offer various services to the users. It mainly has a user interface to enable the user to interact with the system with minimal efforts.

In our case it is the means by which users interact with the devices remotely.

\section{WEB INTERFACE}

The communication between a user and software which is running on web server is known as web interfacing. The user interface is the web browser and the web page it downloaded and rendered.

\section{POWER SUPPLY}

This unit is the provider of the crucial power to all the devices for operation without any overload or damage. Power supply is connected to the home environment because that is where the switchboard is. Non-fluctuating power supply is the basic requirement for operation of the system.

\section{HOME ENVIRONMENT}

Environment of a system is the surroundings in which it operates. In our system there is a fixed home environment which is accessed using the remote system. The home environment consists of the microcontroller, relays, LEDs and power supply. This is basically our switchbox and its components attached to the old switchbox of the system.

\section{REMOTE SYSTEM}

Remote system is used for accessing the system when user is not in the local vicinity. Remote system in our case consists of a mobile application (android) and a web interface. Remote system interacts with home environment via internet.

\section{INTERNET}

Internet is the global system of interconnected network of computers that employ internet protocol suite to link ' $n$ ' number of devices universally. In our project internet is the main component because without internet there is no way for interaction to take place between the android application and devices controlled by the switchbox.

Description of other components used for implementation of the IOT Based Switchbox is given below in much detail:

- For remote access, the application makes use of wifi and an extremely light weight protocol called MQTT protocol which will be described in the next section.

- Also, in order to control the relays, provide wifi connectivity and communicate the software commands to the hardware we require a CC3200 launchpad described later in the paper.

- The launchpad in turn is connected to the relay driving circuit providing voltage for operation of the entire system.

\section{1) MQTT-MQ Telemetry Transport}

MQTT is a Machine-to-machine (M2M)/"internet of things" connectivity protocol.

Some of its features are: 
- Exceptionally lightweight publish / subscribe messaging transport

- Used on top of TCP/IP protocol stack

- Useful for providing connectivity with remote locations

- Fundamentally useful for constrained devices and unreliable networks with low-bandwidth and/or highlatency.

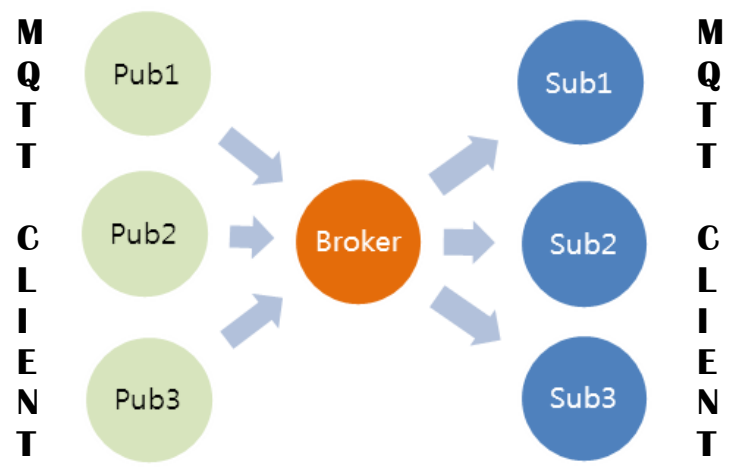

MQTT client-

Fig. MQTT Client and Broker

Any device having a MQTT library running and is connecting to an MQTT broker over any kind of network is a MQTT client.

Types of MQTT clients-
- Publisher- client who is currently sends a particular message

- Subscriber - client (or clients) attempting to receive the message

MQTT broker-

A MQTT broker performs following tasks:

- Receives and filters messages.

- Sends the message to all subscribed clients.

- Provides authentication and authorization of clients.

\section{MQTT topics}

MQTT topics are used for determinations of which message gets to which client and hence effectively used for message filtering and routing.

\section{2) CC3200 LAUNCHPAD}

Features of CC3200 LaunchPad are as follows

- programmable wifi controller

- single chip wireless mcu with built-in wifi

- cortex-m4 MCU for developing an entire application with a single IC

- micro usb connector for power and debug

The CC3200 LaunchPad contains components as shown in the following diagram:

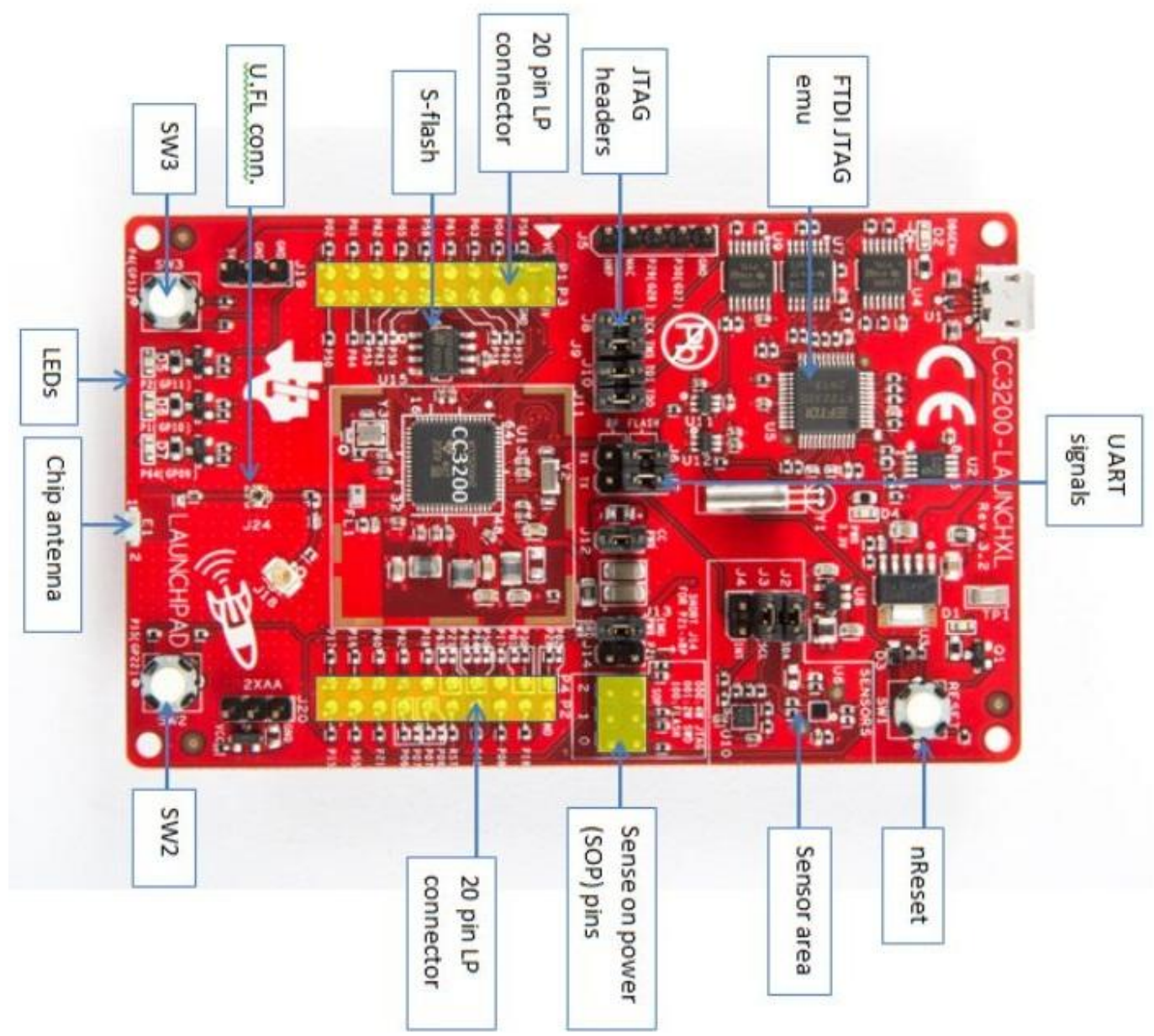

Fig. CC3200 Launchpad 


\section{MATHEMATICAL MODEL}

A mathematical model is a description of a system using concepts of mathematics and language. A model is helpful for explaining of a system and studying the effects of different components. It can also be used to make predictions about behavior.

\section{Mathematical model from overall system}

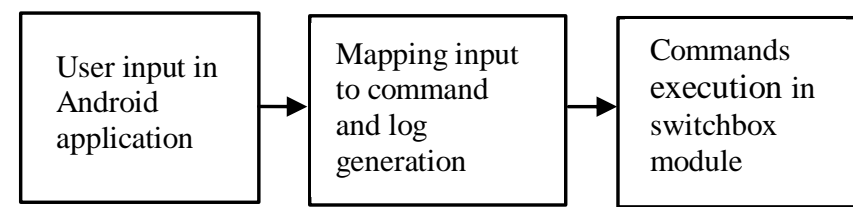

INPUT
$\mathrm{S}=\{\mathbf{I}, \mathbf{O}, \mathbf{F}, \mathbf{F c}, \mathrm{Sc}\}$
I : Set of inputs.
$\mathbf{O}:$ Set of outputs.
F : Set of functions.
Fc : Set of failure cases.
Sc : Set of success cases.

Input :

- Command Received from that Application.

Processing : Addresses resolved for appropriate module, routed accordingly.

Output :

- Appliance 1 switched on/off.

- Appliance 'n' switched on/off.

- Appliance $1 \mathrm{dimmed} / \mathrm{sharpened}$.

- Appliance 'n' dimmed/sharpened.

Functions :

- On/Off

- Dim/Sharpen

Failure cases :

- Address resolved incorrectly.

- Hardware non-responsive.

Success cases : Command successfully executed by Module.

\section{Mathematical model for application}

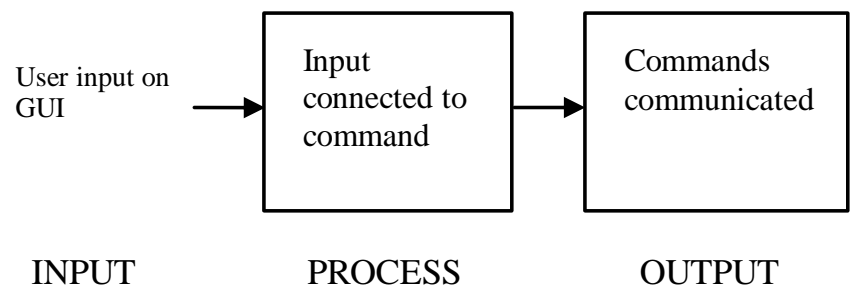

The complete system is comprised of sets of I, O, F, Fc, Sc.

$$
\begin{aligned}
& \mathbf{S}=\{\mathbf{I}, \mathbf{O}, \mathbf{F}, \mathbf{F c}, \mathbf{S c}\} \\
& \mathbf{I}: \text { Set of inputs. } \\
& \mathbf{O}: \text { Set of outputs. } \\
& \mathbf{F}: \text { : Set of functions. } \\
& \mathbf{F c}: \text { Set of failure cases. } \\
& \text { Sc }: \text { Set of success cases. }
\end{aligned}
$$

\section{Input :}

- Appliance 1 switch on/off.

- Appliance ' $n$ ' switch on/off.

- Appliance $1 \mathrm{dim} / \mathrm{sharpen}$.

- Appliance 'n' dim/sharpen.

Processing : Command communicated successfully.

\section{Output :}

- Appliance 1 switch on/off Command Generated .

- Appliance 'n' switch on/off Command Generated.

- Appliance $1 \mathrm{dim} / \mathrm{sharp}$ Command Generated.

- Appliance 'n' dim/sharp Command Generated. 
Functions :

- On/Off

- Dim/Sharpen

$\bullet$

Failure cases :

- Command mapped inaccurately.

Success cases :

- Command sent and acknowledged successfully.

\section{Mathematical model for analytics}

\begin{tabular}{|c|c|c|}
\hline $\begin{array}{l}\text { Log file } \\
\text { from the } \\
\text { modules }\end{array}$ & $\begin{array}{l}\text { Analytics } \\
\text { engine } \\
\text { recognizes } \\
\text { similarities } \\
\text { and patterns }\end{array}$ & $\begin{array}{l}\text { Modification/ } \\
\text { improvement } \\
\text { made } \\
\text { accordingly }\end{array}$ \\
\hline INPUT & PROCESS & OUTPUT \\
\hline
\end{tabular}

The complete system is comprised of sets of I, O, F, Fc, Sc.

$\mathrm{S}=\{\mathrm{I}, \mathrm{O}, \mathrm{F}, \mathrm{Fc}, \mathrm{Sc}\}$

I : Set of inputs.

$\mathbf{O}$ : Set of outputs.

F : Set of functions.

Fc : Set of failure cases.

Sc : Set of success cases.

\section{Input :}

- Log text collected together.

Processing : Pattern Recognition and matching for better features.

\section{Output :}

- Bug fixes.

- Improvements and additional features implemented.

- Improved usability.

Failure cases :

- Log misunderstood, misrepresented.

Success cases : Analytics developed and features developed successfully.

\section{LITERATURE SURVEY}

\section{INTERNET OF THINGS}

Internet Of Things (IoT) is the network of physical "things" embedded with electronics, software, sensors, and connectivity for enabling objects to trade data with the operator and/or other connected devices based on infrastructure of ITUGSI. IoT allows objects to be sensed and controlled remotely across existing infrastructure of network. This in turn ends up creating opportunities for more direct integration between the computer-based systems and the physical world. Hence, resulting in improvement in efficiency, accuracy and economic benefit. Each "thing" is not only distinctively identifiable through its embedded system of computers but is also able to interoperate within the existing internet infrastructure.

Things, in Internet Of Things, can refer to a wide variety of devices such as heart monitoring implants, automobiles with built-in sensors, or field operation devices that assist firefighters in search and rescue.

These devices collect valuable data with the help of various technologies currently existing (eg sensors of various kinds) and then autonomously send the data to other connected devices.

\section{HOME AUTOMATION}

Home automation refers to using computers and IT for controlling home appliances (example lights). It generally includes centralized control of lighting, HVAC, appliances, security, locks of gates and doors and other systems, to provide improved convenience, comfort, energy efficiency and security. Devices may be connected through a home network to allow control by a PC, and remote access through the internet. Systems can range from simple to complex computer/micro-controller based networks. In simple installations, automation may be as straightforward as turning on the lights when a person enters a room. In advanced installations, rooms can sense not only presence of a person inside but know who that person is and perhaps set appropriate lighting, temperature, music etc taking into account day of the week, time of the day and other factors.

System elements- 1. Sensors 2. Controllers 3. Actuators 4. Buses 5. Interfaces

\section{HOME AUTOMATION USING IOT}

IoT devices can be used to monitor and control the mechanical and electronic devices/gadgets used in various buildings (e.g. public and private, industrial, institutions, or residential). Home automation systems, like other building automation systems, are typically used to control lighting, heating, ventilation, air conditioning, appliances, communication systems, entertainment and home security. This in turns improves convenience, comfort, energy efficiency, and security. Home automation and home security is an important advancement when it comes to applying IoT. With IoT, we can control the electrical devices installed in the house while working at the office. Everything connected with the help of internet.

Papers referred and conclusion drawn from those papers:

\section{An Android Multi-protocol Application for} Heterogeneous Building Automation Systems

Application: Building automation- dimmers, switch controls for hvac etc

Advantage: Useful for heterogeneous components

Uses auto-generated xml file

Multi-protocol middleware

Disadvantage: No way to integrate the existing components 
2. A Location-aware Architecture for Heterogeneous Building Automation Systems

Applications: Smart Buildings example, hospital for disabled persons

Advantage: Can handle heterogenous devices and support future devices

Based on Bluetooth low energy

Works on user-defined rules and location

Disadvantage: Explicit commands and implicit rule preference conflict

Security issues

\section{An IoT-based User-centric Ecosystem for Heterogeneous} Smart Home Environments

Advantages: Implementation of machine learning, preemptive scheduling, gesture profile mapping, event prioritization and security policies, medium access control lightweight discovery and message exchange protocol disadvantage: design interference resilient protocols modeling of aggregate interference is critical

\section{Enabling IoT Empowered Smart Lighting Solutions: A Communication Theoretic Perspective \\ Applications: Smart homes \\ Advantages: Localisation mechanism based services \\ New services, applications autonomously \\ Multi-protocol middleware \\ Transparent, heterogeneous access \\ Hides lowlevel communication details}

Disadvantages: Explicit commands and implicit rule preference conflictSecurity issues

\section{Smart Home System based on IOT Technologies}

Applications: Smart home with interactive components

Advantages: Highly scalable

Uses rfid

Uses certification method

Existing components can be used by adding rfid tag

Disadvantages: Security issues

Issue for memoryless components

\section{CONCLUSION}

IoT enables the interaction between people and things at virtual level. IOT and Internet will soon become an integral the part of human life.

From all the above sections, we can summarize that the IOT based SwitchBox has many benefits which are listed below:

1. It is compatible with current household appliances. Meaning that there is no need to discard current technologies, instead we need to collaborate with them to provide a better life.

2. It is modular and scalable. This means that any new appliance which complies with this architecture and protocols can be added into the system

3. It is convenient to use this system because the whole system is controllable remotely by the user via an application. This application is runnable on any device which supporting android. The GUI is quite informative and easy to use and hence there is not much learning requirement for the user to run this application.

Hence, the proposed system can bring about a major change in the IoT based projects. It can play a major role in enhancing our current lifestyle and enabling ease of access by reducing human efforts for basic tasks.

\section{ACKNOWLEDGEMENT}

We would like to express deep sense of gratitude to Prof. Suja Panicker, Maharashtra Institute of Technology, Pune for providing us with her help whenever required. We would also like to thank our family and friends for their continuous support.

\section{REFERENCES}

[1] An Android Multi-protocol Application for Heterogeneous Building Automation Systems Luca Mainetti, Vincenzo Mighali, Luigi Patrono Dept. of Innovation Engineering University of Salento Lecce, ITALY, Spftware Telecommunications and computer networks,2014 International conference.

[2] Smart Home System based on IOT Technologies Yin Jie, Ji Yong Pei, Li Jun, Guo Yun, Xu Wei Department of High Technology Shenzhen Academy of Inspection and Quarantine Shenzhen, China, 2013 International Conference on Computational and Information Sciences

[3] A Location-aware Architecture for Heterogeneous Building Automation Systems Luca Mainetti, Vincenzo Mighali, Luigi Patrono Dept. of Innovation Engineering University of Salento Lecce, ITALY, 2015 IFIP/IEEE International Symposium on Integrated Network Management (IM2015): Experience Session Paper1066

[4] Enabling IoT Empowered Smart Lighting Solutions: A Communication Theoretic Perspective Syed Ali Raza Zaidi, Member, IEEE, Ali Imran, Member,IEEE, Des. C. McLernon, Member,IEEE, and Mounir Ghogho, Senior Member, IEEE, IEEE WCNC 2014- Workshop on IoT Communications and Technology

[5] An IoT-based User-centric Ecosystem for Heterogeneous Smart Home Environments Luca Mainetti, Vincenzo Mighali, Luigi Patrono Dept. of Innovation Engineering University of Salento Lecce, ITALY, IEEE ICC 2015 SAC-Internet of Things

[6] Sharing User IoT Devices in the Cloud Yazid BENAZZOUZ, Christophe MUNILLA, Ozan GNALP, Mathieu GALLISSOT, Levent GRGEN CEA-LETI Minatec, Grenoble, France, 2014 IEEE World Forum on Internet of Things (WF-IoT)

[7] Wireless Sensor Networks for Internet of Things, Nacer Khalil , Mohamed Riduan Abid, Driss Benhaddou , Michael Gerndt IEEE Ninth International Conference on Intelligent Sensors, Sensor Networks and Information Processing (ISSNIP) Symposium on Public Internet of Things Singapore, 2124 April 2014

[8] Securing private wireless sensors in a shared environment in the internet of things context Anass RGHIOUI*1, Said BOUCHKAREN2, Anass KHANNOUS1, Mohammed BOUHORMA1 1LIST FSTT 
(Laboratory of Informatics, Systems and

Telecommunications) 2LabTIC ENSAT (Laboratory of

Technology of Information and Communication)

Abdelmalek Essaadi University Tangier, Morocco , IEEE

2014

[9] Balancing power consumption in IoT devices by using variable packet size M. Domingo-Prieto, B. Martinez, M. Monton, I. Vilajosana-Guillen, X. Vilajosana-Guillen and J. Arnedo-Moreno Internet Interdisciplinary Institute (IN3), Universitat Oberta de Catalunya C/ Roc Boronat,117, 7 our 08018 Barcelona, Spain Email: mdomingopr@uoc.edu Computer Science, Multimedia and Telecomunication Department, Universitat Oberta de Catalunya Rambla del Poblenou 156, 08018 Barcelona, Spain Email: jarnedo, xvilajosana@uoc.edu WorldSensing, C/ Arago 383, 4th our, 08013, Barcelona, Spain, 2014 Eighth International Conference on Complex, Intelligent and Software Intensive Systems

[10] IoT for smart home, IEEE conference 2013 